\title{
General Intomology/Intomologia Geral Ocorrência e caracterização de galhas entomógenas em um fragmento florestal em estágio de sucessão ecológica na Amazônia
}

\author{
Adenomar Neves de Carvalho ${ }^{\bowtie}$ \& Juliana Santos da Mota
}

Universidade Federal do Oeste do Pará - UFOPA.

\section{EntomoBrasilis 11 (2): 118-123 (2018)}

Resumo. O estudo objetivou registrar e caracterizar as galhas entomógenas em duas áreas de floresta em sucessão ecológica em Santarém-PA, de setembro de 2014 a fevereiro de 2015: Campus Tapajós e Bosque Mekdece. Foram coletadas 405 galhas distribuídas em nove morfotipos, os quais estavam associados a 21 espécies de plantas hospedeiras distribuídas em 11 famílias. As plantas hospedeiras que apresentaram maior riqueza de galhas foram Fabaceae com oito morfotipos, Myrtaceae com quatro, seguida de Anacardiaceae e Meliaceae com três e dois morfotipos respectivamente. As folhas foram o órgão vegetal mais galhado (89\%) e as formas globóides as mais frequentes. Os Cecidomyiidae (Diptera) foram os principais cecidógenos e os parasitóides encontrados pertenciam às famílias Eulophidae, Encyrtidae e Trichogramatidae (Hymenoptera).

Palavras-chave: Biodiversidade; Cecídea; Cecidomyiidae; Interação inseto-planta; Plantas hospedeiras.

\section{Occurrence and characterization of entomogenous galls in a forest fragment at the stage of ecological succession in the Amazon}

Abstract. The study aimed to record and characterize the entomogenic galls in two forest areas in ecological succession in Santarém-PA, from September 2014 to February 2015: Campus Tapajós and Mekdece Forest. The total of 405 gallons were collected in nine morphotypes, which were associated with 21 host plant species distributed in 11 families. The host plants that presented the greatest richness of galls were Fabaceae with eight morphotypes, Myrtaceae with four, followed by Anacardiaceae and Meliaceae with three and two morphotypes, respectively. The leaves were the most scattered plant organ (89\%) and the most frequent globose forms. The Cecidomyiidae (Diptera) were the main cecidogens and the parasitoids found belonged to the families Eulophidae, Encyrtidae and Trichogramatidae (Hymenoptera).

Keywords: Biodiversity; Cecídea; Cecidomyiidae; Host plants; Insect-plant interaction.

会 s galhas são o resultado de hiperplasia e hipertrofia celular induzida em plantas por diferentes organismos, a maioria deles insetos, como Diptera, Hymenoptera e Coleoptera (Toma \& MENDonçA Júnior 2013), as quais servem de incubadoras para os insetos em desenvolvimento de onde obtém nutrição e proteção contra condições abióticas desfavoráveis e inimigos naturais (PRICE et al. 1987). Há indícios que o inseto controla o seu desenvolvimento, porém, este controle está sujeito à adequação e reatividade dos tecidos da planta hospedeira (KurZFELD-ZeXer et al. 2015), porém, o mecanismo indutor permanece desconhecido. De certa forma a indução de galhas representa uma adaptação do inseto à planta hospedeira, bem como uma adaptação da planta ao inseto (Oliveira et al. 2016).

O conhecimento da riqueza de insetos galhadores e suas plantas hospedeiras é importante para o entendimento e determinação de padrões de distribuição deste grupo de herbívoros. Embora os estudos enfocando insetos galhadores aumentaram significativamente nas últimas décadas, principalmente no Brasil, a maioria dos biomas foi investigado apenas uma única vez (Fernandes et al. 2004; JULĩ̃o et al. 2005; MAIA et al. 2008; Coelho et al. 2009; SANTos et al. 2010; Almada \& FERnANDES 2011; ARAÚJo et al. 2011).

Assim, o oprincipal objetivo deste estudo é apresentar dados de morfotipos de galhas induzidas por insetos e suas respectivas plantas hospedeiras em um fragmento florestal em estágio inicial de sucessão ecológica em Bioma Amazônico no oeste paraense.

\section{MATERIAL E MÉTODOS}

O estudo foi realizado em uma área de reflorestamento e sucessão ecológica de 1,4 hectares, denominado Bosque Mekdece. A restauração florestal do bosque ocorreu com a reintrodução de espécies nativas da Amazônia e seu progresso atualmente está associado ao recrutamento do banco de sementes, assim como dos processos de dispersão zoocórica e anemocórica. A vegetação arbórea do Campus Tapajós da Universidade Federal do Oeste do Pará, em Santarém-PA ( $02^{\circ} 25^{\prime}$ S e $54^{\circ} 44^{\prime}$ W), com uma área de 12 hectares, também foram amostradas. Através da conceituação fisionômica-ecológica do RADAMBRASIL

\section{Edited by:}

Rodrigo Souza Santos

Article History:

Received: $27 . i v .2018$

Accepted: 23.vii.2018

\section{Corresponding author:}

Adenomar Neves de Carvalho

○adenomarc@yahoo.com.br

(3) http://orcid.org/0000-0002-4354-488X

\section{Funding agencies:}

$\checkmark$ Fundação Amazônia de Amparo a Estudos e Pesquisas - FAPESPA 
(1976), a vegetação local (original), foi denominada de Floresta Tropical Aberta, caracterizada por florestas com lianas e várias espécies de palmeiras. Segundo Veloso et al. (1991) estas florestas apresentam um aspecto degradado, onde as copas não se tocam.

O clima dominantemente da região é quente e úmido, característico das florestas tropicais, com temperatura média anual variando de 25 a $28^{\circ} \mathrm{C}$. A precipitação pluvial média anual é de $1.920 \mathrm{~mm}$, com maior intensidade no período de dezembro a maio, e menor intensidade nos meses de junho a novembro correspondente ao período mais seco (INMET 2017).

As coletas foram realizadas mensalmente de setembro de 2014 a fevereiro de 2015, totalizando sete coletas. Em cada área foi realizada uma amostragem de uma hora por amostragem (totalizando sete horas/área amostrada $=14 \mathrm{~h}$ ). A cada amostragem, durante caminhadas de $1 \mathrm{~h}$, todas as plantas com até $5 \mathrm{~m}$ de altura foram vistoriadas com um binóculo quanto à presença de galhas.

As plantas que apresentaram galhas foram coletadas com o auxílio de podão com cabo telescópico para identificá-las e caracterizar o inseto galhador, como sugerido por PRICE et al. (1998). Sempre que possível, as plantas hospedeiras foram identificadas por especialistas até o nível específico.

Em laboratório, sob estereomicroscópio, as galhas foram fotografadas e em seguida dissecadas para obter as formas imaturas dos insetos galhadores, bem como descrever os principais aspectos morfológicos das mesmas, tais como: forma, cor, número de câmaras e presença de tricomas.

Morfotipos de galhas foram utilizados como um indicador de espécies de galhadores porque praticamente todas as espécies de insetos galhadores amazônicos são novas para a ciência (ALmADA \& Fernandes 2011). Segundo Price et al. (1998), assim como CARNEIro et al. (2009), a utilização de morfotipos de galhas, associado ao órgão vegetal de ocorrência, são indicadores confiáveis da espécie de insetos indutores, dada sua morfologia única e alta especificidade à planta hospedeira e ao órgão da planta atacado.

Os insetos galhadores adultos foram obtidos a partir da criação, mantendo amostras de cada morfotipo de galha individualmente em frascos de plástico cobertos com tecido voil e um chumaço de algodão embebido com água no fundo. Esses frascos foram examinados diariamente para verificar a emergência dos adultos e possíveis inimigos naturais, os quais foram identificados até o nível taxonômico de ordem e família com o uso de literatura especializada (RAFAEL et al. 2012). Todos os insetos coletados foram preservados em etanol a 70\% e estão depositados no Laboratório de Entomologia da Universidade Federal do Oeste do Pará (UFOPA).

\section{RESULTADOS}

Foram coletadas, ao todo, 405 galhas de insetos em 27 árvores distribuídas em 11 famílias no bosque Mekdece e Campus Tapajós (Tabela 1). Dentre as espécies de árvores identificadas, 10 espécies ocorreram no bosque e 17 espécies no Campus Tapajós. Ao todo foram amostradas nove morfotipos insetos indutores de galhas, sendo cinco morfotipos no bosque e nove morfotipos no Campus Tapajós, sendo que destes, quatro morfotipos ocorreram exclusivamente neste ambiente (Figuras 1 e 2). Além dos cecidomiídeos, foram obtidos vários parasitoides, todos himenópteros, os quais serão futuramente identificados ao nível de espécie.

Tabela 1. Caracterização morfológica das galhas encontradas no Bosque Mekdece e Campus Tapajós da Universidade Federal do Oeste do Pará, Santarém-PA. Legenda: Órgão ( F = Folha; R = Ramo), Superfície (Amb = Ambas, Abx = Abaxial, Adx = Adaxial), Morfotipo (Disc $=$ Discóide, Cil = Cilíndrica, Elip = Elíptica, $\mathrm{Glb}=$ Globoide, Esf $=$ Esférica, Con $=$ Cônica, Ovoide $=$ Ovo, Bif $=$ Bífida, Par = Parenquimática), Tricomas ( N = Não, S = Sim), Câmaras (Var = Várias) e NI = Não identificada.

\begin{tabular}{|c|c|c|c|c|c|c|c|c|c|c|}
\hline Planta hospedeira & $\begin{array}{l}\text { No de } \\
\text { Galhas }\end{array}$ & Órgãos & Superficie & Morfotipo & $\begin{array}{c}\mathrm{N}^{\circ} \text { de } \\
\text { Câmaras }\end{array}$ & Cor & Tricoma & $\begin{array}{l}\text { Galhador } \\
\text { (Família) }\end{array}$ & $\begin{array}{l}\text { Parasitoide } \\
\text { (Família) }\end{array}$ & Local \\
\hline
\end{tabular}

Myrtaceae

\begin{tabular}{|c|c|c|c|c|c|c|c|c|c|c|}
\hline Myrcia spheciosa & 2 & $\mathrm{~F}$ & $\mathrm{Amb}$ & Esf & Var & Verde & $\mathrm{N}$ & Cecidomyiidae & - & Campus Tapajós \\
\hline Myrcia spheciosa & 1 & $\mathrm{~F}$ & $\mathrm{Amb}$ & Glb & 1 & Verde & $\mathrm{N}$ & Cecidomyiidae & Eulophidae & Campus Tapajós \\
\hline Myrcia bracteata & 3 & $\mathrm{~F}$ & $\mathrm{Amb}$ & Glb & 1 & Verde & $\mathrm{N}$ & Cecidomyiidae & Eulophidae & Campus Tapajós \\
\hline Myrcia bracteata & 1 & $\mathrm{~F}$ & $\mathrm{Amb}$ & Ovo & 1 & Vermelha & $\mathrm{N}$ & Cecidomyiidae & Eulophidae & Campus Tapajós \\
\hline Myrcia bracteata & 1 & $\mathrm{~F}$ & $\mathrm{Amb}$ & Bif & 1 & Verde & $\mathrm{N}$ & Cecidomyiidae & Eulophidae & Campus Tapajós \\
\hline
\end{tabular}

Mimosaceae

$\begin{array}{lllllllll}\text { Inga sp. } & 1 & \mathrm{~F} & \mathrm{Amb} & \mathrm{Glb} & 1 & \text { Verde } & \mathrm{N} & \text { NI }\end{array}$

NI

Campus Tapajós

Burseraceae

$\begin{array}{lllllllll}\text { Protium sp. } & 2 & \text { F/R } & \text { Abx } & \text { Esf } & 1 & \text { Vermelha } & \text { N } & \text { Phlaeothripidae }\end{array}$

Campus Tapajós

Meliaceae

\begin{tabular}{|c|c|c|c|c|c|c|c|c|c|c|}
\hline Swietenia macrophyll & 1 & $\mathrm{~F}$ & Adx & Ovo & 1 & Marrom & $\mathrm{N}$ & Cecidomyiidae & Eulophidae & Campus Tapajós \\
\hline Carapa guianensis. & 1 & $\mathrm{~F}$ & Adx & Elip & 1 & Verde & $\mathrm{N}$ & Cecidomyiidae & Encyrtidae & Campus Tapajós \\
\hline \multicolumn{11}{|l|}{ Moraceae } \\
\hline Bagassa guianensis & 1 & $\mathrm{~F}$ & $\mathrm{Abx}$ & Ovo & 1 & Marrom & $\mathrm{N}$ & Cecidomyiidae & Trichogrammatidade & Bosque Mekdece \\
\hline \multicolumn{11}{|l|}{ Fabaceae } \\
\hline Copaifera multijuga & 1 & $\mathrm{~F}$ & $\mathrm{Amb}$ & Bif & Var & Marrom & $\mathrm{N}$ & Cecidomyiidae & Eulophidae & Campus Tapajós \\
\hline Copaifera multijuga & 1 & $\mathrm{~F}$ & $\mathrm{Amb}$ & Con & 1 & Marrom & $\mathrm{N}$ & Cecidomyiidae & Eulophidae & Campus Tapajós \\
\hline
\end{tabular}




\begin{tabular}{|c|c|c|c|c|c|c|c|c|c|c|}
\hline Planta hospedeira & $\begin{array}{l}\mathrm{N}^{\circ} \text { de } \\
\text { Galhas }\end{array}$ & Órgãos & Superfície & Morfotipo & $\begin{array}{c}\mathrm{N}^{\circ} \text { de } \\
\text { Câmaras }\end{array}$ & Cor & Tricoma & $\begin{array}{l}\text { Galhador } \\
\text { (Família) }\end{array}$ & $\begin{array}{l}\text { Parasitoide } \\
\text { (Família) }\end{array}$ & Local \\
\hline Copaifera multijuga & 3 & $\mathrm{~F}$ & $\mathrm{Amb}$ & Disc & 1 & Marrom & $\mathrm{N}$ & Cecidomyiidae & Eulophidae & Campus Tapajós \\
\hline Bowdichia sp. & 1 & $\mathrm{~F}$ & Abx & Elip & 1 & Verde & $\mathrm{N}$ & Cecidomyiidae & Eulophidae & Campus Tapajós \\
\hline $\begin{array}{l}\text { Macrolobium } \\
\text { microcalyx }\end{array}$ & 1 & $\mathrm{~F}$ & $\mathrm{Amb}$ & Par & 1 & Marrom & $\mathrm{N}$ & Cecidomyiidae & Eulophidae & Bosque Mekdece \\
\hline Bauhinea sp. & 1 & $\mathrm{~F}$ & $\operatorname{Adx}$ & Elip & 1 & Marrom & S & Cecidomyiidae & Eulophidae & Bosque Mekdece \\
\hline Hymenaea courbaril. & 1 & $\mathrm{~F}$ & $\mathrm{Amb}$ & $\mathrm{Glb}$ & 1 & Marrom & $\mathrm{N}$ & Cecidomyiidae & Eulophidae & Campus Tapajós \\
\hline Swartzia apetala & 1 & $\mathrm{~F}$ & $\operatorname{Adx}$ & Esf & 1 & Verde & $\mathrm{N}$ & Cecidomyiidae & Eulophidae & Bosque Mekdece \\
\hline Swartzia apetala & 2 & $\mathrm{~F}$ & $\operatorname{Adx}$ & Elip & 1 & Verde & $\mathrm{N}$ & Cecidomyiidae & Encyrtidae & Bosque Mekdece \\
\hline \multicolumn{11}{|l|}{ Melastomatacea } \\
\hline Miconia guianensis & 1 & $\mathrm{~F}$ & $\operatorname{Adx}$ & $\mathrm{Glb}$ & 1 & Verde & $\mathrm{N}$ & Cecidomyiidae & Eulophidae & Bosque Mekdece \\
\hline \multicolumn{11}{|l|}{ Piperaceae } \\
\hline $\begin{array}{l}\text { Pothomorphe } \\
\text { umbellata }\end{array}$ & 1 & $\mathrm{~F}$ & Abx & Par & 1 & Marrom & $\mathrm{S}$ & NI & - & Campus Tapajós \\
\hline \multicolumn{11}{|l|}{ Sapotaceae } \\
\hline Pouteria guianensis & 2 & $\mathrm{~F}$ & $\mathrm{Amb}$ & Par & 1 & Marrom & $\mathrm{S}$ & Cecidomyiidae & Eulophidae & Campus Tapajós \\
\hline \multicolumn{11}{|l|}{ Anacardiaceae } \\
\hline $\begin{array}{l}\text { Anacardium } \\
\text { occidentale }\end{array}$ & 1 & $\mathrm{~F}$ & $\operatorname{Adx}$ & Cil & 1 & Verde & $\mathrm{N}$ & NI & - & Campus Tapajós \\
\hline Astronium gracile & 2 & $\mathrm{~F} / \mathrm{R}$ & $\mathrm{Abx}$ & Par & Var & Verde & $\mathrm{N}$ & Cecidomyiidae & Eulophidae & Bosque Mekdece \\
\hline Spondias mombin & 2 & $\mathrm{~F} / \mathrm{R}$ & $\mathrm{Abx}$ & Par & 1 & Marrom & $\mathrm{S}$ & NI & - & Bosque Mekdece \\
\hline Tapirira guianensis & 1 & $\mathrm{~F}$ & $\operatorname{Adx}$ & Elip & 1 & Verde & $\mathrm{N}$ & NI & - & Bosque Mekdece \\
\hline \multicolumn{11}{|l|}{ Bignoniaceae } \\
\hline Tabebuia serratifolia & 1 & $\mathrm{~F}$ & $\operatorname{Adx}$ & $\mathrm{Glb}$ & Var & Marrom & $\mathrm{N}$ & Cecidomyiidae & Trichogrammatidae & Bosque Mekdece \\
\hline
\end{tabular}

A quase totalidade das galhas foram induzidas por espécies de Diptera (77,8\%), seguida por Thysanoptera (3,7\%) e por galhadores não identificados $(18,5 \%)$. Quanto ao órgão vegetal afetado, a maioria das galhas foram induzidas exclusivamente nas folhas $(88,9 \%)$, enquanto $11,1 \%$ ocorreram tanto nas folhas quanto nos ramos. Grande parte das galhas eram glabras $(85,2 \%)$ e somente $14,8 \%$ apresentaram tricomas. Quanto ao número de câmaras $85,2 \%$ tinham uma única câmara e 14,8\% de duas a três. As galhas globoides foram a forma mais comum encontrada (22,2\%), seguida pelas elípticas e parenquimáticas (18,5\% cada), esféricas e ovoides (11,1\% cada) e bífidas (7,4\%) (Figuras 1 e 2). As formas cônicas, discoides e cilíndricas juntas somaram 11,1\%. Em relação à cor, as galhas verdes foram as mais abundantes $(48,2 \%)$, seguida pelas marrons $(44,4 \%)$. Foram encontradas apenas duas galhas vermelhas $(7,4 \%)$.

As famílias mais representativas de plantas hospedeiras foram Fabaceae com oito morfotipos (29,6\%), Myrtaceae com quatro $(14,8 \%)$, Anacardiaceae com três $(11,1 \%)$ e Meliaceae com dois $(7,4 \%)$, totalizando mais da metade dos morfotipos encontrados $(63 \%)$.

\section{DISCUSSÃO}

JULĩ̃o (2007) encontrou 275 morfotipos de galhas em 14 fragmentos de floresta de terra firme a $70 \mathrm{~km}$ ao norte de Manaus-AM, totalizando 117 hectares. AlmadA \& FERnANDEs (2011) reportou 309 morfotipos de galhas em áreas de reflorestamento da Mineração Rio do Norte em Porto Trombetas, distrito de Oriximiná-PA. O presente estudo identificou nove morfotipos, aparentemente pouco, quando comparado aos estudos realizados no mesmo bioma. No entanto, deve-se considerar que tais estudos foram realizados em áreas de florestas não antropizadas, além da grande diferença nas áreas amostradas e nos esforços amostrais entre as pesquisas comparadas.
O táxon de inseto galhador predominante foi Cecidomyiidae (Diptera), assim como os registrados em diversos outros estudos (Espírito-Santo \& Fernandes 2007; Toma \& Mendonça Júnior 2013). Esse resultado corrobora a hipótese de que Cecidomyiidae é o grupo taxonômico mais especializado de insetos galhadores na região Neotropical (GAGNÉ 2010). Além de Diptera, também foi constatado a ocorrência de galhas induzidas por Thysanoptera (Phlaeothripidae) em Protium sp. (Myrtaceae).

É importante ressaltar que houveram muitos insetos galhadores não identificados (18,5\%) oriundos de plantas com poucas galhas, que devido ao intenso ataque de parasitoides inviabilizaram a emergência dos galhadores adultos.

Notamos que houve neste estudo uma forte predominância de ocorrência de galhas nas folhas, fato comum para galhas de insetos (Fernandes et al. 1988; Dreger-JaufFret \& Shorthouse 1992; Maia \& Fernandes 2004; Moreira et al. 2007 e Maia et al. 2008).

A predominância de galhas glabras foi observada no presente estudo. Porém, segundo Toma \& Mendonça Júnior (2013) esse fato segue um padrão entre insetos galhadores e tem sido amplamente relatado por outros pesquisadores (EzCURRA et al. 1987; Toma \& MENDONÇA JÚNIOR 2013).

No presente trabalho encontramos uma grande ocorrência de galhas isoladas. Esse fato pode ser explicado pela hipótese levantada por FERNANDEs et al. (1988) que afirmam que as galhas isoladas seriam favorecidas porque a pressão exercida pelos parasitoides seria diminuída devido ao maior tempo de busca imposto pelo isolamento.

Duas famílias de plantas hospedeiras apresentaram maior número de morfotipos de galhas encontradas neste estudo: Fabaceae e Myrtaceae. Myrtaceae figura como táxon importante em outros tipos de vegetação no Sul do Brasil (MENDONÇA et al. 

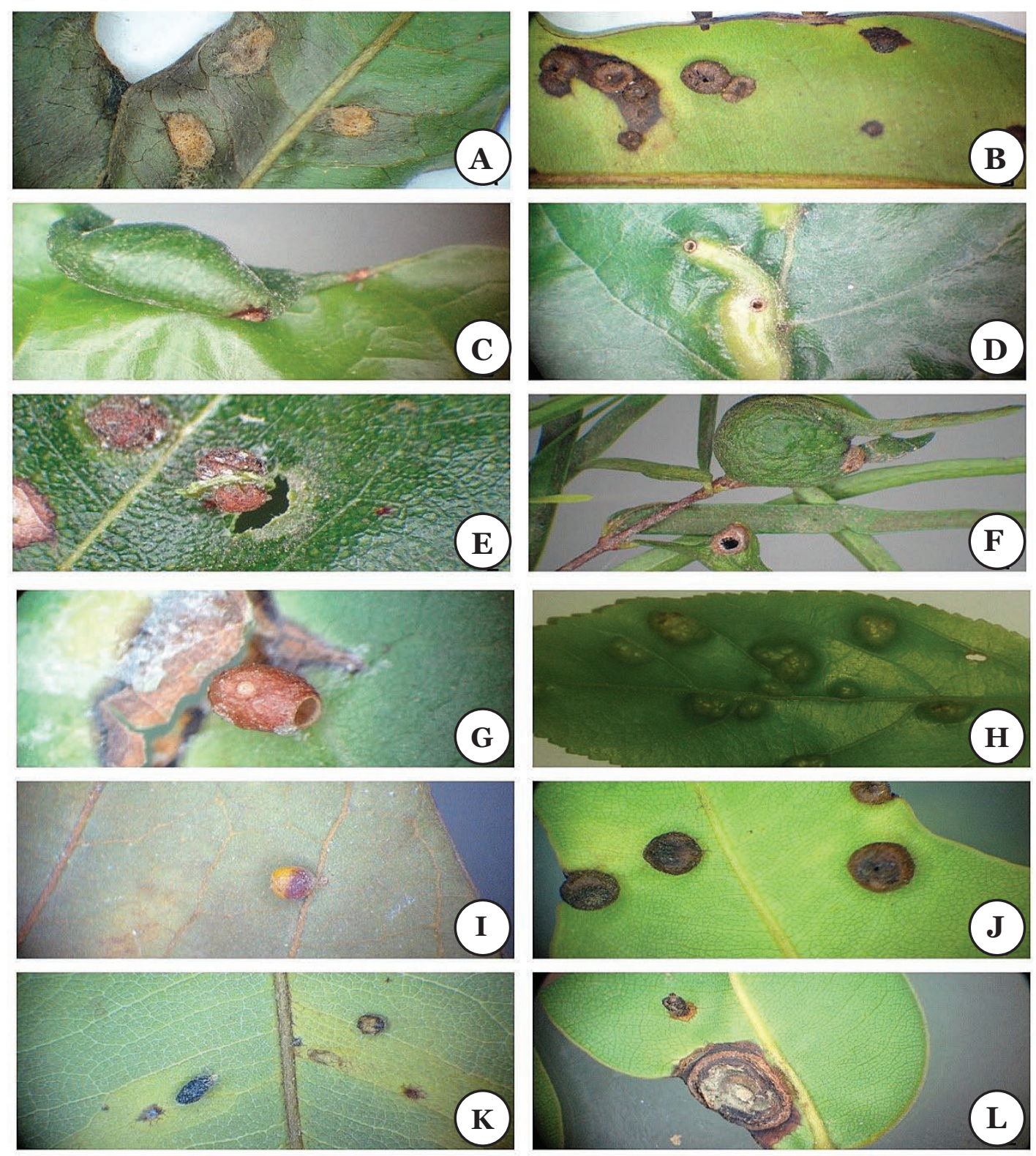

Figura 1. Plantas hospedeiras e morfotipos de galhas encontradas no Bosque Mekdece e Campus Tapajós da Universidade Federal do Oeste do Pará, Santarém-PA. (A) Pouteria guianensis Aubl. (parenquimática); (B) Anacardium occidentale L. (cilíndrica); (C) Carapa guianensis Aubl. (elíptica); (D) Swartzia apetala Lindl. (elíptica); (E) Hymenaea courbaril L. (globoide); (F) Myrcia spheciosa (Amshoff) McVaugh (esférica); (G) Bagassa guianensis Aubl. (ovoide); (H) Astronium gracile Engl. (parenquimática); (I) Miconia guianensis (Aubl.) Cogn. (globoide); (J) Copaifera multijuga Hayne (discoide); (K) Copaifera multijuga Hayne (cônica) e (L) Copaifera multijuga Hayne (bífida).

2010), Cerrado (Gonçalves-Alvim \& Fernandes 2001), Campos Rupestres (MAIA \& FERNANDES 2004) e Restinga (MAIA et al. 2008). Em bioma Amazônico, Almada \& Fernandes (2011) pesquisando na Floresta Nacional do Saracá Taquera (Porto Trombetas, distrito de Oriximiná-PA), concluiu que a família Fabaceae foi a principal hospedeira, resultado idêntico ao encontrado no presente estudo.

Este é o primeiro estudo de galhas e seu indutores em área de sucessão florestal na Amazônia e corrobora a hipótese de que Cecidomyiidae é o principal indutor de galhas na região Neotropical. Os dados amostrados também indicam uma possível preferência por plantas hospedeiras da família Fabaceae em bioma amazônico. Em geral, as características das galhas encontradas neste estudo acompanham um padrão já relatado para o neotrópico, provavelmente refletindo uma correlação espacial em uma escala biogeográfica maior.

Segundo Price (1991) insetos galhadores geralmente estão associados a ambientes onde predominam vegetações escleromórficas. Esse tipo devegetação é caracterizado por possuir baixas probabilidades de abcisão de folhas, folhas perenes e com altas concentrações de fenóis (Fernandes \& Price 1991). Outros estudos também verificaram que galhas de insetos têm baixas probabilidades de sobreviver em habitats úmidos comparado a habitats xéricos, que restringem a ocupação de organismos por suas condições de umidade e temperatura (Juliño 2007). Nestes habitats, insetos galhadores podem apresentar maiores taxas de parasitismo, bem como de predação (Fernandes et al. 2005). De certa forma, a maior riqueza de insetos galhadores encontrados no Campus Tapajós corrobora tal hipótese, por possuir uma vegetação mais aberta, portanto menos úmida, representam nichos relativamente livres de patógenos e de inimigos naturais, como parasitoides e herbívoros que se alimentam de galhas devido à sua alta concentração de nutrientes.

Mais estudos sobre insetos indutores de galhas em áreas de sucessão ecológica na Amazônia devem ser realizados, a fim de fornecer uma visão mais clara de sua biodiversidade.

\section{AGRADECIMENTOS}

Os autores agradecem ao Instituto de Biodiversidade e Florestas pelo apoio à pesquisa e à UFOPA pela concessão da bolsa de iniciação científica ao segundo autor. 

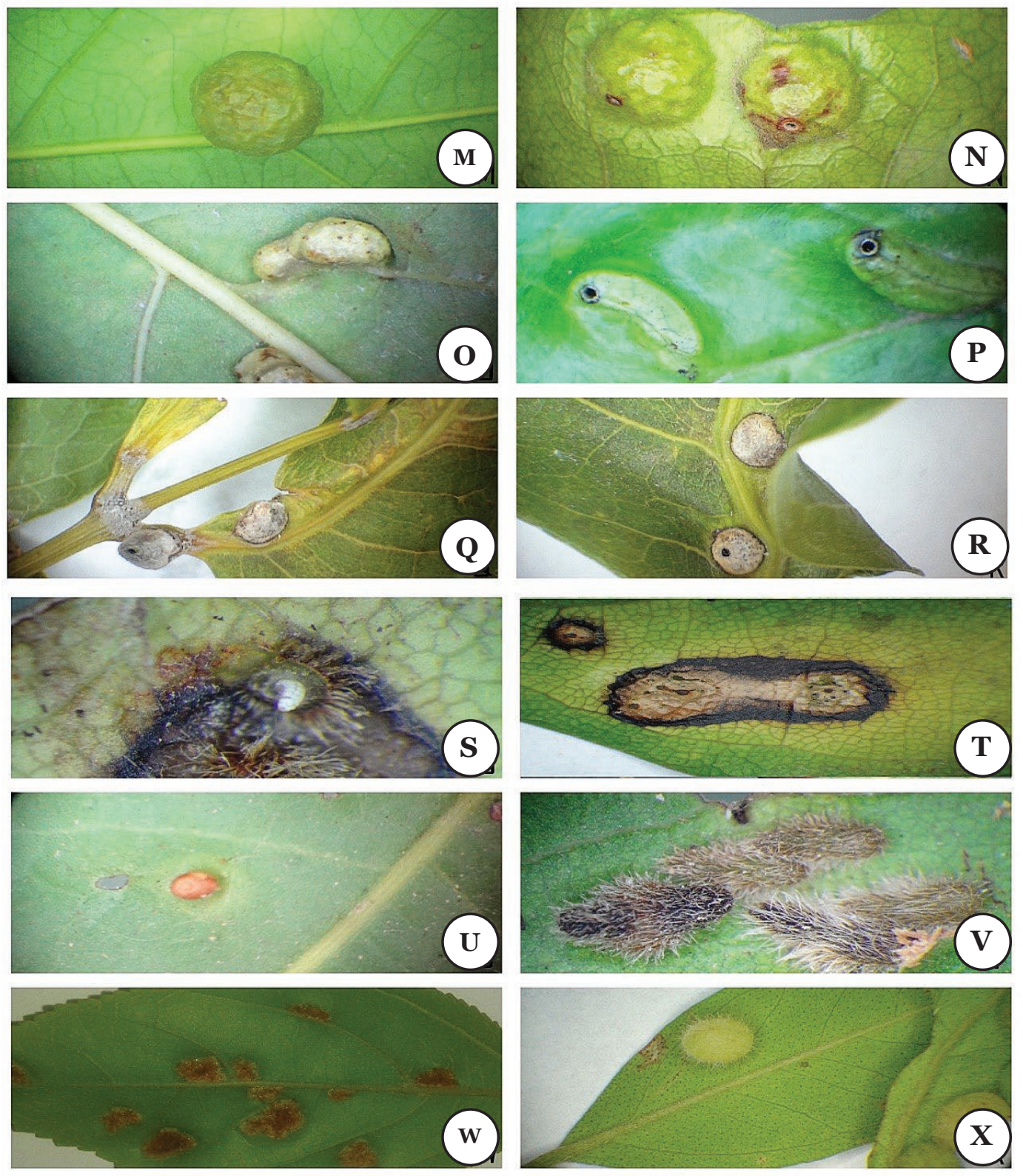

Figura 2. Plantas hospedeiras e morfotipos de galhas encontradas no Bosque Mekdece e Campus Tapajós da Universidade Federal do Oeste do Pará, Santarém-PA. (M) Inga sp. (globoide); (N) Myrcia bracteata (Rich.) DC. (globoide); (O) Bowdichia sp. Kunth (elíptica); (P) Tapirira guianensis Aubl. (elíptica); (Q) Protium sp. Burm.f. (esférica); (R) Protium sp. Burm.f. (Cônica); (S) Pothomorphe umbellata (L.) Miq. (Parenquimática); (T) Macrolobium microcalyx Ducke (parenquimática); (U) Tabebuia serratifolia (Vahl) G. Nicholson (globoide); (V) Bauhinea sp. L. (elíptica); (W) Spondias mombin L. (parenquimática) e (X) Myrcia spheciosa (Amshoff) McVaugh (globoide).

\section{REFERÊNCIAS}

Almada, E.D. \& G.W. Fernandes, 2011. Insetos indutores de galhas em florestas de terra firme e em reflorestamentos com espécies nativas na Amazônia Oriental, Pará, Brasil. Boletim do Museu Paraense Emilio Goeldi, 6: 163-196.

Araújo, W.S., B.B. Santos \& V.L. Gomes-Klein, 2011. Insect galls from Serra dos Pireneus, GO, Brazil. Biota Neotropica, 11: 357-365. DOI: https://doi.org/10.1590/s167606032011000200034.

Carneiro, M.A.A, C.S.A. Branco, C.E.D. Braga, E.D. Almada, M.B.M. Costa, E.G. Fernandes \& V.C. Maia, 2009. Are gall midge species (Diptera: Cecidomyiidae) host plant specialists? Revista Brasileira de Entomologia, 53: 365-378. DOI: https://doi.org/10.1590/s0085-56262009000300010.

Coelho, M.S., E.D. Almada, G.W. Fernandes, M.A.A. Carneiro, R.M. Santos, A.V. Quintino \& A. Sanchez-Azofeifa, 2009. Gall inducing arthropods from a seasonally dry tropical Forest in Serra do Cipó, Brazil. Revista Brasileira de Entomologia, 53: 404-414. DOI: https://doi.org/10.1590/s008556262009000300015 .

Dreger-Jauffret, F. \& J.D. Shorthouse, 1992. Diversity of gallinducing insects and their galls, p. 8-33. In: Shorthouse, J.D.
\& O. Rohfritsch (Eds.). Biology of insect-induced galls. New York, Oxford University Press, xi+285 p.

Espírito-Santo, M.M. \& G.W. Fernandes, 2007. How many species of gall-inducing insects are there on earth, and where are they? Annals of the Entomological Society of America, 100: 95-99.

Ezcurra, E., J.C. Gomez \& J. Becerra, 1987. Diverging patterns of host use by phytophagous insects in relation to leaf pubescence in Arbutus xalapensis (Ericaceae). Oecologia, 72: 479-8o. DOI: https://doi.org/10.1007/bfoo377583.

Fernandes, G.W. \& P.W. Price, 1991. Comparisons of tropical and temperate galling species richness: the role of environmental harshness and plant nutrient status, p. 91-116. In: Price, P.W., T.M. Lewinsohn, G. W. Fernandes \& W.W. Benson (Eds.). Plant-animal interactions: evolutionary ecology in tropical and temperate regions. John Wiley, New York. ooo p.

Fernandes, G.W., E. Tameirão-Neto \& R.P. Martins, 1988. Ocorrência e caracterização de galhas entomógenas no Campus-Pampulha da UFMG. Revista Brasileira de Zoologia, 5: 11-29. DOI: https://doi.org/10.1590/s010181751988000100002.

Fernandes, G.W., F.M.C. Castro, M.L. Faria, E.S.A. Marques \& M.K.B. Greco, 2004. Effects of hygrothermal stress, plant 
richness and architecture on mine insect diversity. Biotropica, 36: 240-247. DOI: https://doi.org/10.1646/99184-q1563.

Fernandes, G.W., S.J. Gonçalves-Alvim \& M.A.A. Carneiro, 2005. Habitat-driven effects on the diversity of gall-inducing insects in the Brazilian cerrado, p. 693-708. In: Raman, A., C.W. Schaefer \& T.M. Withers (Eds.). Biology, ecology, and evolution of gall-inducing arthropods. Science Publishers, Enfield (NH), USA. oooo p.

Gagné, R.J., 2010. Update for a catalog of the Cecidomyiidae (Diptera) of the world. Disponível em: <https://www.ars. usda.gov/ARSUserFiles/12754100/gagne 2010 world catalog cecidomyiidae.pdf $>$. [Acesso em: 26.iv.2018].

Gonçalves-Alvim, S.J. \& G.W. Fernandes, 2001. Biodiversity of galling insects: historical, community and habitat effect in four neotropical savannas. Biodiversity and Conservation, 10: 79-98.

INMET- Instituto Nacional de Meteorologia, 2017. Disponível em INMET- Instituto Nacional de Meteorologia. Disponível em: <http://www.inmet.gov.br/>. [Acesso em: 26.iv.2018].

Julião, G.R., 2007. Riqueza e abundância de insetos galhadores associados ao dossel de florestas de terra firme, várzea e igapó da Amazônia Central. Tese (Doutorado em Ecologia). Instituto Nacional de Pesquisas da Amazônia. $158 \mathrm{f}$.

Kurzfeld-Zexer, L., S. Lev-Yadun \& M. Inbar, 2015. One aphid species induces three gall types on a single plant: Comparative histology of one genotype and multiple extended phenotypes. Flora - Morphology, Distribution, Functional Ecology of Plants, 210: 19-30. DOI: https://doi.org/10.1016/j.flora.2014.10.007.

Maia, V.C. \& G.W. Fernandes, 2004. Insect galls from Serra de São José (Tiradentes, MG, Brazil). Brazilian Journal of Biology, 64: 423-445. DOI: https://doi.org/10.159o/s151969842004000300007 .

Maia, V.C., M.A.G. Magenta \& S.E. Martins, 2008. Ocorrência e caracterização de galhas de insetos em áreas de restinga de Bertioga (São Paulo, Brasil). Biota Neotropica, 8: 167-197. DOI: https://doi.org/10.1590/s1676-06032008000100020.

Mendonça, M.S., H.M.F. Piccardi, S.M. Jahnke \& R.V. Dalbem, 2010. Galling arthropod diversity in adjacent swamp forests and restinga vegetation in Rio Grande do Sul, Brazil. Neotropical Entomology, 39: 513-518. DOI: https://doi.org/10.1590/s1519-566x2010000400008.
Moreira, R.G., G.W. Fernandes, E.D. Almada \& J.C. Santos, 2007. Galling insects as bioindicators of land restoration in an area of Brazilian Atlantic Forest. Lundiana, 8: 107-112.

Oliveira, D.C., R.M.S. Isaias, G.W. Fernandes, B.G. Ferreira, R.G.S. Carneiro \& L. Fuzaro, 2016. Manipulation of host plant cells and tissues by gall-inducing insects and adaptive strategies used by different feeding guilds. Journal of Insect Physiology, 84: 103-113. DOI: https://doi.org/10.1016/j. jinsphys.2015.11.012.

Price, P.W., 1991. Patterns in communities along latitudinal gradients, p. 51-70. In: Price, P.W., T.M. Lewinsohn, G.W. Fernandes \& W.W. Benson (Eds.), Plant-animal interactions: Evolutionary ecology in tropical and temperate regions. New York, John Wiley, ooo p.

Price, P.W., G.W. Fernandes \& G.L. Waring, 1987. Adaptive nature of insect galls. Environment Entomology, 16: 15-24. DOI: https://doi.org/10.1093/ee/16.1.15.

Price, P.W., G.W. Fernandes, A.C. Lara, J. Bran, H. Barrios, M.G. Right, S.P. Ribeiro \& N. Rotcliff, 1998. Global patterns in local number of insect galling species. Journal of Biogeographi, 25: 581-591. DOI: https://doi.org/10.1046/ j.1365-2699.1998.2530581.x

RADAMBRASIL, 1976. Departamento Nacional de Produção Mineral. Folha AS.21- Santarém. Geologia, geomorfologia, pedologia, vegetação e uso potencial da terra. (Levantamento dos Recursos Naturais, v. 10). Rio de Janeiro: DNPM, 510 p.

Rafael, J.A., G.A.R. Melo, C.J.B. Carvalho, S.A. Casari \& R. Constatino, 2012. Insetos do Brasil: Diversidade e Taxonomia. Holos Editora, Ribeirão Preto, SP. 810 p.

Santos, B.B., H.D. Ferreira \& W.S. Araújo, 2010. Ocorrência e caracterização de galhas entomógenas em uma área de floresta estacional semidecídua em Goiânia, Goiás, Brasil. Acta Botanica Brasilica, 24: 243-249. DOI: https://doi.org/10.1590/s0102-33062010000100026.

Toma, T.S.P. \& M.S. Mendonça Júnior, 2013. Gall-inducing insects of an Araucaria Forest in southern Brazil. Revista Brasileira de Entomologia, 57: 225-233. DOI: https://doi.org/10.1590/s0085-56262013005000001.

Veloso, H.P.,A.L.R. Rangel Filho \& J.C.A. Lima, 1991. Classificação da vegetação brasileira, adaptada a um sistema universal. Instituto Brasileiro de Geografia e Estatística - IBGE. Rio de Janeiro, Brasil. 123 p.

\section{Suggestion citation:}

Carvalho, A.N. \& J.S. Mota, 2018. Ocorrência e caracterização de galhas entomógenas em um fragmento florestal em estágio de sucessão ecológica na Amazônia. EntomoBrasilis, 11 (2): 118-123.

Available on: doi:10.12741/ebrasilis.v11i2.786
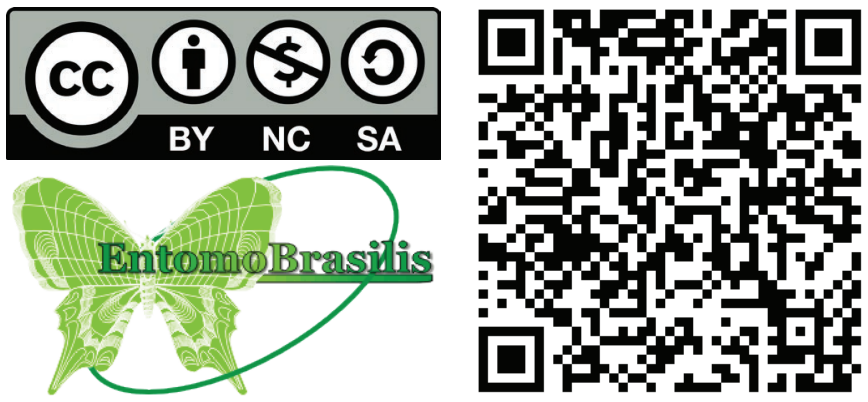